\title{
A study of remote plasma enhanced CVD of silicon nitride films
}

\author{
S.E. ALEXANDROV ${ }^{(1)}$, M.L. HITCHMAN and S. SHAMLIAN \\ Department of Pure and Applied Chemistry, University of Strathclyde, 295 Cathedral Street, Glasgow \\ G1 1XL, United Kingdon
}

\begin{abstract}
The growth of silicon nitride ( $\mathrm{SiNxHy}$ ) films from $\mathrm{SiH}_{4}$ and $\mathrm{N}_{2}$ by capacitively coupled remote PECVD is described for the first time. The influence of process parameters on the growth rate, concentration of bonded hydrogen, and properties of deposited films is discussed. The most probable mechanism of film formation is proposed on the basis of the experimental results obtained.
\end{abstract}

\section{Introduction}

Thin silicon nitride films (SiNxHy) deposited at relatively low temperatures $200-400^{\circ} \mathrm{C}$ have found widespread use in integrated circuit technology as interlevel dielectrics, encapsulation films, gate dielectrics, diffiasion barriers, and as final passivation layers [1,2]. Among a number of different low temperature methods of deposition of dielectrics, the most widely used commercial method and the most intensively studied process is that of plasma enhanced chemical vapour deposition (PECVD) [3]. The majority of PECVD systems have been based on the use of RF capacitively coupled, parallel plate electrodes with a glow discharge in an appropriate gas mixture being formed between them. The substrates are usually placed on the surface of one of the electrodes. This conventional PECVD approach has several disadvantages, the main ones being :

- electrically active defects are likely to be generated near the surface of the semiconductor substrate due to bombardment by energetic particles during the deposition process.

- plasma bombardment of the growing dielectric film may cause generation of pinholes and the appearance of charge in the film.

- complex reactions can be induced due to the simultaneous production of many sorts of reacting species, some of which may participate in the film formation process and this mechanism leads to deposition of films with unpredictable composition.

- process parameters such as the total pressure, RF-power, gas composition, gas flow rate etc. are all interdependent and difficult to control individually.

Much of the research in the field of PECVD of dielectric films in general and of silicon based dielectrics, such as silicon nitride, in particular, has been directed towards overcoming some of these problems [4]. One approach is to generate the plasma away from the substrate, so that there is less likelihood of plasma bombardment of the growing film. In this technique, known as remote PECVD (RPECVD), RF power is usually coupled into the system inductively for excitation of the nitrogen-containing initial reagents. Silane, along with a noble gas diluent, is introduced downstream from the discharge region because of the ease with which it can be decomposed in the plasma [5]. 
There appears to have been no studies reported on the use of capacitive coupling of RF power into a remote glow discharge. It therefore seemed to us to be interesting to investigate the use of RPECVD with capacitive coupling, especially since, if it was successful, it should be possible to use the technique with relatively minor modification in industrial PECVD reactors.

\section{Experimental}

A schematic diagram of the experimental reaction chamber with the capacitively coupled RF power used in this work is shown in Figure 1. The glow discharge was generated by RF excitation between the two circular parallel plate electrodes, which were separated by a $4 \mathrm{~cm}$ gap and were positioned in the upper part of the reaction chamber with the lower electrode being $16 \mathrm{~cm}$ above the substrate on the heating platform. The upper, grounded electrode consisted of a stainless steel plate (diameter $36 \mathrm{~cm}$ ) with a central region (diameter $4 \mathrm{~cm}$ ) with holes (diameter $0.12 \mathrm{~mm}$ ) which acted as a gas distributor. The lower, powered electrode (diameter $20 \mathrm{~cm}$ ) was made from a stainless steel mesh and was connected to an RF power generator (Yaesu FT-757QX) which operated at $13.56 \mathrm{MHz}$ through an antenna tuner (Yaesu FC-902).

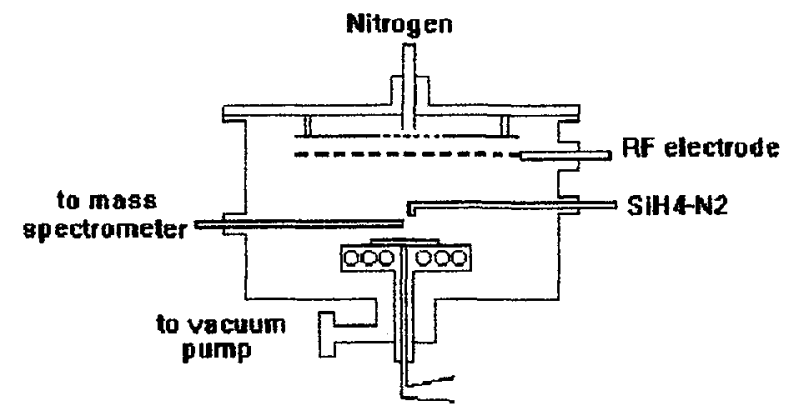

Fig.1. Schematic diagram of the remote PECVD reactor.

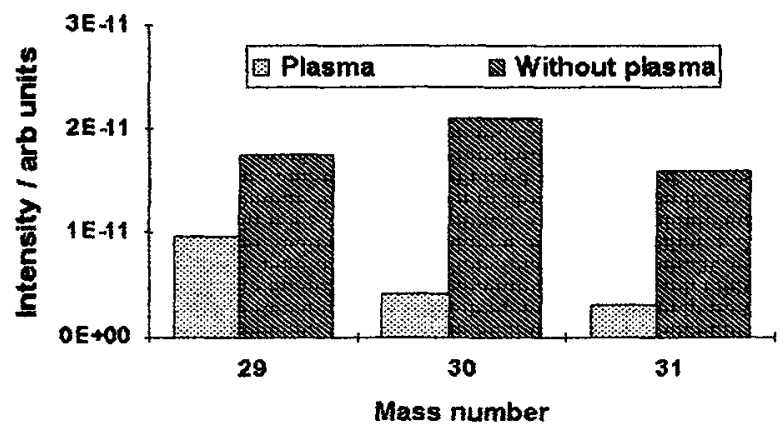

Fig. 2. A typical mass spectrum in the vicinity of $\mathrm{SiH}_{\mathrm{X}}{ }^{+}$peaks.

As indicated in Figure 1, the reactant gases, electronic grade nitrogen and silane, were introduced into the stainless steel reaction chamber (diameter $38 \mathrm{~cm}$ ) separately. The flow rate of nitrogen $\left(\mathrm{QN}^{\mathrm{t}}\right.$ ) through the showerhead arrangement in the upper electrode was varied in the range $50-150 \mathrm{sccm}$. The silane/nitrogen mixture was introduced from the side of the reaction chamber downstream from the discharge region 
through an injection nozzle positioned $6 \mathrm{~cm}$ above the substrate. The silane flow $\left(\mathrm{QS}_{\mathrm{S}}\right)$ was in the range 0.2 to $50 \mathrm{sccm}$ and the nitrogen flow $\left(\mathrm{QN}^{\mathrm{S}}\right)$ in the range $5-10 \mathrm{sccm}$; this gave a silane to total nitrogen flow ratio in the range $1.3^{*} 10^{-3}$ to $90.9^{*} 10^{-3}$. These gas fiow conditions minimised backstreaming of the silane in the direction of the plasma electrode and also gave high uniformity of layer growth, with a typical variation being less than $2 \%$ over the entire surface of a $3 "$ wafer.

One of the vacuum ports in the side of the deposition chamber was used to link the reactor to a quadrapole mass spectrometer (VG XPS 600). The ioniser of this spectrometer was connected to the port with a stainless steel tube of diameter $1 / 8^{\prime \prime}$ and of length $60 \mathrm{~cm}$; the geometry of the equipment prevented a closer positioning of the ioniser to the reaction zone.

Film thickness and refractive index were measured using an automatic ellipsometer (Gaertner L116B) with a He-Ne laser. FTIR transmission spectra of the layers were obtained with a Galaxy Series FTIR 3000 spectrometer. The number density of $\mathrm{Si}-\mathrm{H}$ and $\mathrm{N}-\mathrm{H}$ bonds was calculated from FTIR spectra using a method given by Lanford and Rand [6].

\section{Experimental Results and Discussion.}

At an early stage of this research, mass-spectrometric analysis of the gas phase during remote PECVD was carried out in order to try and get a better understanding of the plasma chemistry.

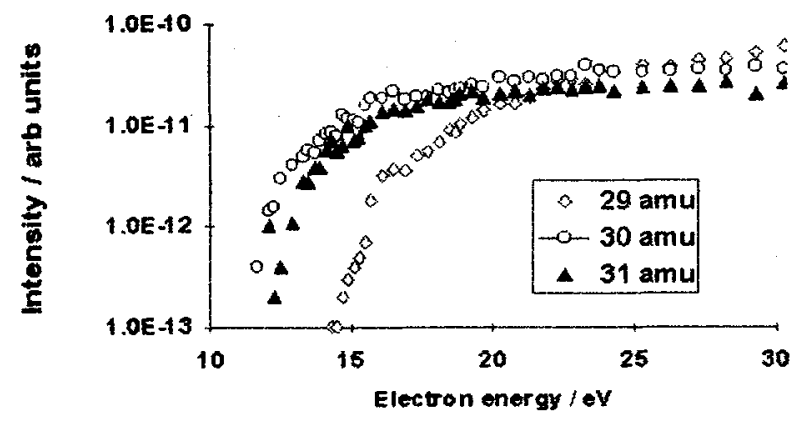

Fig. 3 Dependence of signal intensity of 29-31 amu peaks on electron ionising energy.

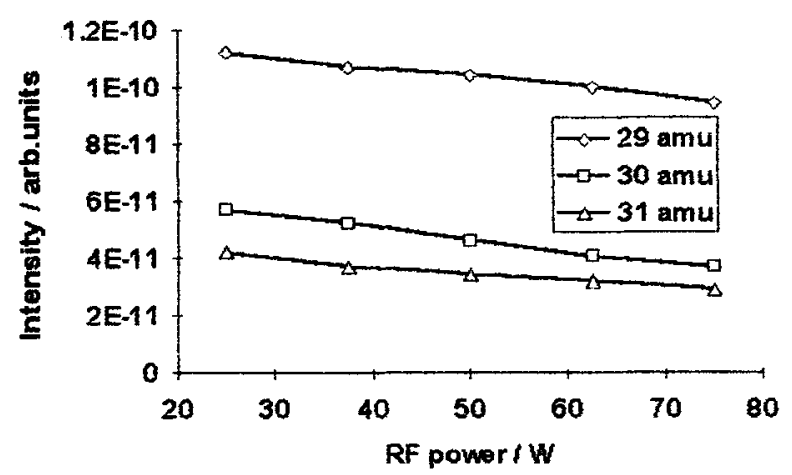

Fig.4. Dependence of signal intensity of 29-31 amu peaks on RF power. 
Figure 2 shows a typical mass-spectrum in the vicinity of the silane radical peaks for process parameters under, what will be seen below to be, preferred deposition conditions of high RF power (75$90 \mathrm{~W}$ ), low silane gas flow rate $(0.2-0.5 \mathrm{sccm})$, and relatively low total pressure $(15-35 \mathrm{~Pa})$. Under these conditions, apart from these peaks and mass peaks corresponding to nitrogen, hydrogen, and residual gases no other peaks have been noticed. Silane is generally difficult to study by mass spectroscopy because its dissociation energy is less than the ionisation energy [7]. Figure 3 shows the dependence of intensity of mass peaks from 29 to 31 amu on the electron ionising energy. From these data the following values of appearance potential for species, corresponding to mass peaks from 29 to 31 , are found: $\sim 15.4 \mathrm{eV}, \sim 12.0 \mathrm{eV}$, and $\sim 12.3 \mathrm{eV}$, respectively. Analysis of the data obtained when the plasma was turned off gave the same values of the appearance potential for mass peaks in the region $29-31 \mathrm{amu}$. These values are in good agreement with the appearance potentials for $\mathrm{SiHx}^{+}(\mathrm{x}=1-3)$ ions created from silane by electron impact ionisation [7,8]. Thus one can conclude that mass peaks from 29 to 31 amu in both cases corresponded to cracking patterns of neutral silane, which was fragmented and ionised in the mass spectrometer. The intensity of all mass peaks corresponding to the silane fragments decreased with increasing RF power (Fig.4). At an RF power of $75 \mathrm{~W}$ the intensity of the 30 and $31 \mathrm{amu}$ mass peaks at the electron energy for impact ionisation of $70 \mathrm{eV}$ was only about $1 / 5$ of that found when the plasma was turned off (See Fig. 2). This indicates that $\sim 80 \%$ of the original silane introduced into the reaction chamber was dissociated into various $\operatorname{SiHx}(x=1-3)$ species due to dissociation processes in the after glow region during remote plasma enhanced CVD. It should be noted that direct mass-spectrometric observation of SiHx species in these experiments was not possible, probably because of the low sensitivity of the mass spectrometer at low energy of ionising electrons (8-14eV), which it was necessary to use for such measurements. For example, we have only found that the weak signal corresponding to $29 \mathrm{amu}$ appeared at an ionising electron energy of about $14 \mathrm{eV}$ which is less than the appearance potential for $\mathrm{SiH}^{+}$created from silane. Also ignition of the RF discharge caused a variation of the relative intensity of mass peaks corresponding to the silane fragments. When the plasma in the $\mathrm{SiH}_{4}-\mathrm{N}_{2}$ system was turned off the peak corresponding to $\mathrm{SiH}_{2}{ }^{+}$was most abundant, whereas under plasma conditions $\mathrm{SiH}^{+}$was dominant(Fig.2). The results of analysis of mass spectra obtained in $\mathrm{SiH}_{4}{ }^{-}$ $\mathrm{N}_{2}$ and $\mathrm{SiH}_{4}-\mathrm{Ar}$ systems with plasma and without plasma show that the variation of the 29 amu mass peak intensity probably reflects the production of SiHx radicals in the after glow discharge region during remote PECVD due to collisions of neutral molecules of silane with excited nitrogen species and electrons from the discharge region, as well as being due to photodissociative processes. Also mass spectra of the gas phase obtained during PECVD at high flow rates of silane $(>0.5 \mathrm{sccm})$ and total pressures more than $40 \mathrm{~Pa}$ could be used as evidence of the production of silane radicals, because a new group of peaks appeared in the region of 56-60 amu. The intensity of these peaks grew with increase of silane flow rate and total pressure. These peaks correspond to disilane and radicals $\mathrm{Si}_{2} \mathrm{H}_{\mathrm{y}}(\mathrm{y}=1-4)$, which can be produced, as has been reported [7], due to homogeneous reactions in the plasma between neutral molecules of silane and its radicals SiHx. These reactions are probably quite rapid because under these process conditions the intense formation of a yellow brown powder deposited on the cold reactor walls took place and caused a decrease of film growth rate (Fig.5). The FTIR spectrum of the powder showed that it consisted of a material with $\mathrm{Si}-\mathrm{Hx}$ and $\mathrm{Si}-\mathrm{Si}$ bonds, and it was therefore probably a mixture of polysilanes; others have reported a similar formation in PECVD systems[9]

From the mass spectrometric results one can suggest that silane introduced into the reaction chamber is dissociated in the after glow region forming SiHx radicals, which can then be adsorbed on the substrate surface. The results of mass spectrometric analysis show no indication of $\mathrm{Si}-\mathrm{N}$ moieties, such as aminosilanes. So since we have no evidence of species with Si-N bonds being formed in gas phase near the substrate one can also suggest that remote PECVD silicon nitride films are formed by heterogeneous reactions between excited nitrogen particles and SiHx radicals as well as residual neutral silane.

Figure 6 shows the dependence of the film growth rate and refractive index on silane flow rate. Increasing the silane flow rate leads to a steady increase in the deposition rate and to a significant 
change in the refractive index. Near stoichiometric PECVD silicon nitride has a refractive index of

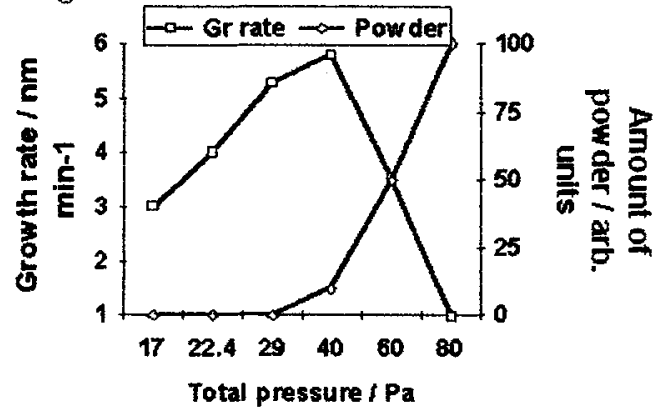

Fig. 5. Dependence of growth rate and amount of powder on total reactor pressure.

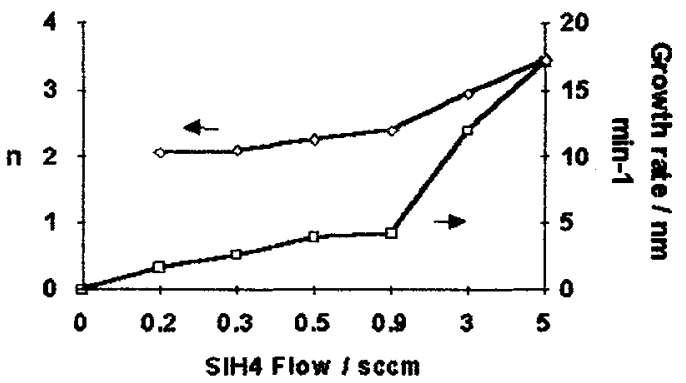

Fig.6. Dependence of growth rate and refractive index on silane flow rate.

$\sim 2.0-2.05$ [2]. So in our system it is necessary to have a total $\mathrm{N}_{2} / \mathrm{SiH}_{4}$ ratio greater than $\sim 250$ in order to deposit films with good stoichiometry. As this gas flow ratio decreases, there is a marked increase in the relative silicon content of the film. This is indicated by a variation of refractive index with silane flow rate and the fact that it is known there is a linear dependence of refractive index on silicon content of a nitride film [10]. The reason why there is such a strong dependence on N/Si ratio in our case is probably because of the limited supply of nitrogen reactive species. For remote PECVD there is a greater chance of loss of the reactive species before they reach the reaction surface. The deficiency of reactive nitrogen species near the substrate surface at a low total $\mathrm{N}_{2} / \mathrm{SiH}_{4}$ ratio leads to an incorporation of SiHx groups in the growing films and causes a concomitant increase in hydrogen uptake in the growing layers. Using the method of Lanford and Rand we have found that for the silicon rich film grown with a N2/ $\mathrm{SiH}_{4}$ ratio of $\sim 30$ the amount of hydrogen preferably bonded to silicon is $\sim 3^{*} 10^{22} \mathrm{~cm}^{-3}$ while for the near stoichiometric film grown with a N2/SiH 4 ratio of $\sim 530$ the hydrogen content is $\sim 1 * 10^{22} \mathrm{~cm}^{-3}$. The amount of hydrogen bonded with nitrogen is $3-8 * 10^{20} \mathrm{~cm}^{-3}$.

It is common for plasma activated processes that the temperature of the substrate has no significant influence on the deposition rate (see Figure 7). The results demonstrate that even though there are some subtle and complex variations of growth rate with temperature between 100 and $350^{\circ} \mathrm{C}$ the total variation from the mean is not more than $+15 \%$. However, the substrate temperature is one of the main factors which determines the film composition, because mobility of active species on the surface of the growing film, rates of bond breaking and rearrangement processes are very strongly influenced by this parameter. Figure 8 shows the variation of film refractive index and concentration of bonded hydrogen in the layers with the deposition temperature. On the basis of these results one can suggest that in the temperature region of about $250-300^{\circ} \mathrm{C}$ the film composition is very close to stoichiometric silicon nitride, containing a relatively small amount of hydrogen. Under those conditions adsorbed active species on the growing surface have enough energy for necessary rearrangements due to both substrate heating and plasma particle bombardment. At lower temperatures $\left(<250^{\circ} \mathrm{C}\right)$ the film growth is probably being controlled by the energy input from the plasma. The fall off in the hydrogen content (see Fig.8) and, in particular, the sharp decrease in refractive index above $200^{\circ} \mathrm{C}$ both indicate a shift away from less dense, silicon rich films $\left(\sim 2.33 \mathrm{~g} / \mathrm{cm}^{3}\right)$ to more dense stoichiometric nitride $\left(\sim 2.8 \mathrm{~g} / \mathrm{cm}^{3}\right)$. A decrease of etch rate in buffered HF and an increase of breakdown strength with substrate temperature also reflect the changes of film hydrogen content and film densification .

Figure 9 shows the variation of the film growth rate with the RF-power, obtained at the different flow rates of nitrogen $(70$ and $150 \mathrm{sccm})$ passed through the plasma generation region. One can see in both cases that the dependencies have a complicated character. However, the absolute variation of the growth rate with RF-power is not significant. At the same time the level of RF-power coupled into the system 


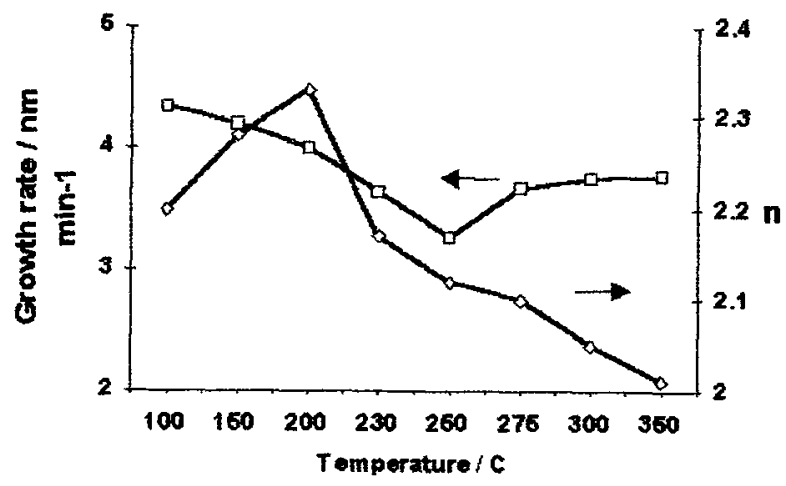

Fig. 7. Dependence of growth rate and refractive index on deposition temperature.

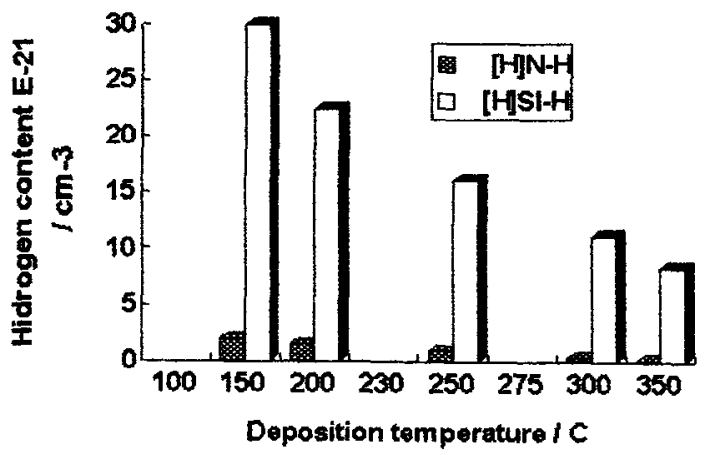

Fig.8. Dependence of bonded hydrogen concentration on deposition temperature.

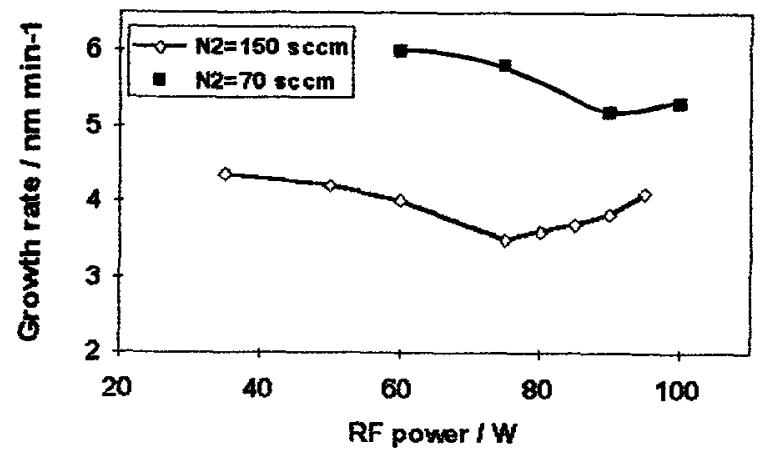

Fig.9. Dependence of growth rate on RF power. 


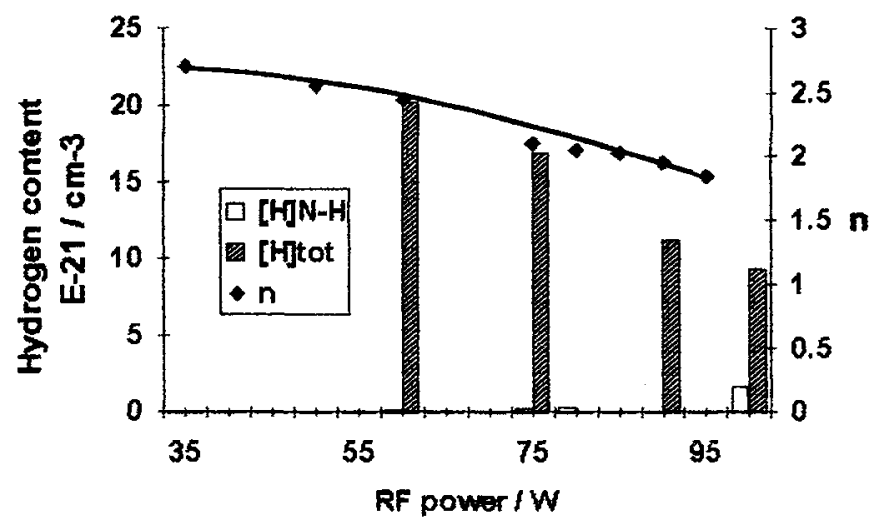

Fig. 10. Dependence of hydrogen content and refractive index on $R F$ power.

must have a very strong influence on the film composition, since without the plasma there is no deposition of silicon nitride films in the $\mathrm{SiH}_{4}-\mathrm{N}_{2}$ system. The gradual change of concentration of hydrogen and of the refractive index (Fig.10) are evidence for this suggestion. Both these dependencies can be explained by a decrease in the amount of energy delivered to the surface of the growing film by excited particles, as well as by a decrease of the concentration of active nitrogen species. An increase in plasma power density should increase the concentration of reactive nitrogen species relative to that of silicon species and so there is a gradual shift of film composition to a near-stoichiometric silicon nitride. In the power region of about 20-80 W with $Q_{N} t=150 \mathrm{sccm}$ a sharp decrease in refractive index from 2.7 to 2.0 has been found. At the same time the deposition rate decreases slightly, probably because of densification of the film. The dependence of etch rate and breakdown strength on RF power also reflects the changes of film hydrogen content and film densification (Fig.11). Values of the refractive index about 2.0 indicate, that the $\mathrm{Si} / \mathrm{N}$ ratio in the deposited films is close to that of near-stoichiometric silicon nitride. With a continued increase in RF power ( $>80 \mathrm{~W}$ ) the densification effect is probably more than compensated for by the increased concentration of active nitrogen species leading to an increase in the rate of growth of a nitrogen rich film. With a decrease in nitrogen flow rate it is necessary to couple into the system an additional amount of $\mathrm{RF}$ power to achieve the deposition of near-stoichiometric silicon nitride films. It is clear that at low flow rates of nitrogen passed through the discharge region the amount of reactive nitrogen species reaching the reaction surface becomes less. It should be noted that the greater growth rate at $\mathrm{QN}^{\mathrm{t}}=70 \mathrm{sccm}$ was due to a larger partial pressure of silane. The formation of $\mathrm{N}-\mathrm{H}$ bonds in the films, probably takes place on the growing surface, as mass spectroscopic analysis of the gas phase shows no indication of species with $\mathrm{N}$ - $\mathrm{Hx}$ bonds, such as has been found earlier in the $\mathrm{SiH}_{4}-\mathrm{NH}_{3} \mathrm{system}$ [11]. Thus one can conclude that formation of near stoichiometric silicon nitride films is possible with process parameters providing a high concentration of reactive nitrogen species in the discharge region and good conditions for their transport to the substrate. The mean value for the rate of silicon nitride deposition ( $3 \mathrm{~mm} / \mathrm{min}$ ) in the system described here under optimum conditions is significantly higher than any reported previously (for example $0.3 \mathrm{~nm} / \mathrm{min}$ [12]) for RPECVD of SiNxHy films with $\mathrm{SiH}_{4}-\mathrm{N}_{2}$ systems. 


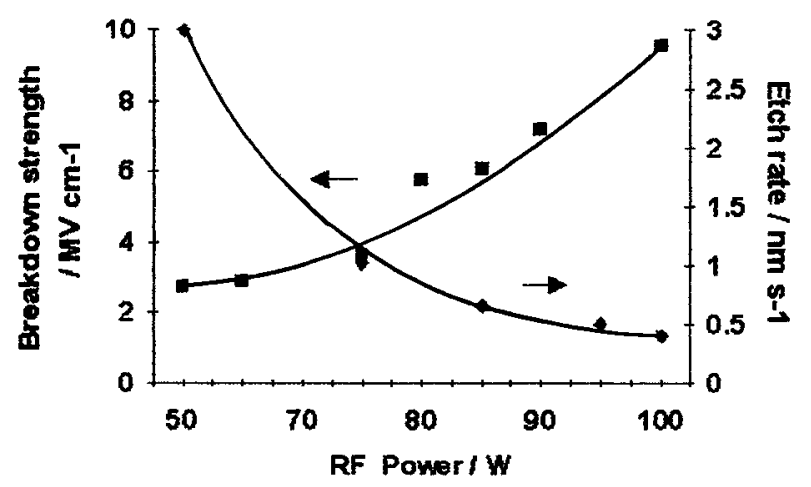

Fig.11 Dependence of breakdown strength and etch rate on $\mathrm{RF}$ power.

\section{Conclusions.}

To summarise, for the mechanism of deposition, we suggest that ground state nitrogen passed through the capacitive coupled discharge region is primarily excited. Excited nitrogen species as well as electrons are transported to the after glow region where they mix with ground state silane. As a result of this mixing active $\mathrm{SiHx}(\mathrm{x}=1-3)$ species are produced in the after glow region and then along with active nitrogen particles arrive at the substrate. The deposition of SiNxHy films is a result of heterogeneous reactions on the growing surface between adsorbed active SiHx radicals and excited nitrogen species. Composition and properties of the deposited films strongly depend on the deposition temperature, gas composition and other process parameters, which determine the concentration of active species as well as amount of energy delivered to the growing surface. Films deposited with near stoichiometric composition contain a relatively low amount of bonded hydrogen and so are of great interest for electronic avolications

\section{References.}

1/ C.R.M. Grosvenor, 'Microelectronic Materials' Adam Hilger, Bristol, 1989.

/2f M. Gupta, V.K. Rathi, R.Thangaraj, O.P. Agnoihotri and K.S. Chari, Thin Solid Films, 1991, 204, 77.

13/ D.W. Hess and D.B. Graves in 'Chemical Vapsour Deposition - Principle and Applications' (ed. M.L. Hitchman and K.F. Jensen), Academic Press, London, 1993, Ch 7.

14/ G. Lucovsky, D.V. Tsu, R.A. Rudder and R.J. Markunas in 'Thin Film Process II' (ed. J.L.Vossen and W.Kern), Academic Press, London, 1991, Ch. IV-2.

/5/ D.V. Tsu, G.N. Parsons, G. Lucovsky and M.W. Watkins, J. Vac. Sci. Technol., 1989, A7, 1115.

16/ W.A.Lanford and M.J.Rand, J. Appl. Phys., 1978, 49, 2473.

17/ H.A. Weakliem in 'Semiconductors and Semimetals v.21A', Academic Press, London, 1984, Ch. 10.

/8/ G. Turban, Y. Catherine and B. Grolleau, Thin Solid Films, 1980, 67, 309.

/9/ D.V. Tsu, G.N. Parsons and G.Lucovsky, J. Vac. Sci. Technol., 1988, A6, 1849.

/10/ W.A.P. Claassen, W.G.J.N. Valkenburg, F.H.P.M. Habraken and Y. Tamminga, J. Electrochem. Soc., 1983, 130, 2419.

11/ D.L. Smith, A.S. Alimonda, C.C. Chen, S.E. Ready and B. Wacker, J. Electrochem. Soc., 1990, 137, 614.

/12/ D. Tsu and G. Lucovsky J. Vac. Sci. Technol., 1986, A4, 480. 